\title{
Variations of the McEliece Cryptosystem
}

\author{
Jessalyn Bolkema, Heide Gluesing-Luerssen, Christine A. Kelley, \\ Kristin Lauter, Beth Malmskog, Joachim Rosenthal *
}

May 1, 2017

\begin{abstract}
Two variations of the McEliece cryptosystem are presented. The first is based on a relaxation of the column permutation in the classical McEliece scrambling process. This is done in such a way that the Hamming weight of the error, added in the encryption process, can be controlled so that efficient decryption remains possible. The second variation is based on the use of spatially coupled moderate-density parity-check codes as secret codes. These codes are known for their excellent error-correction performance and allow for a relatively low key size in the cryptosystem. For both variants the security with respect to known attacks is discussed.
\end{abstract}

\section{Introduction}

Many widely-used public key cryptosystems, such as RSA and elliptic curve cryptography, can be broken by a large-scale quantum computer. It was pointed out in a report by National Institute of Standards and Technology [1] that the building of powerful quantum computers might well be feasible in a matter of 20 years and for this reason the report encourages academics to come up with systems which might still be safe in an environment where quantum computers exist.

One promising family of public key systems which are potentially secure in a postquantum computer environment are so called code-based cryptosystems, like the McEliece [34] and the Niederreiter [40] systems. These systems are based on the hardness of decoding a random linear code, a problem that has so far proved resistant to quantum computer-aided attacks. In both these cryptosystems the secret key is a code for which an efficient decoding algorithm is known. The public key is a disguised version of the secret code that appears to be a random code, thus hiding the structure that gives rise to efficient decoding.

The security of such a system is thus based on two assumptions. First, it is intractable to decode the seemingly random public code with general decoding algorithms. Since the best known algorithms for decoding random linear codes are exponential in the length of the code [33, 7], this can in principle be achieved by increasing the size of the code. However, this comes at the cost of a prohibitively large key size. The second assumption is that it is impossible for an attacker to reconstruct or uncover the underlying structure of the

* Jessalyn Bolkema at University of Nebraska-Lincoln, jessalyn.bolkema@huskers.unl.edu; Heide GluesingLuerssen at University of Kentucky, heide.gl@uky.edu; Christine Kelley at University of Nebraska-Lincoln, ckelley2@math.unl.edu; Kristin Lauter at Microsoft Research, klauter@microsoft.com; Beth Malmskog at Vilanova University, beth.malmskog@gmail.com; Joachim Rosenthal at University of Zürich, rosenthal@math.uzh.ch 
secret code from the public code. This prevents the attacker from simply using the efficient decoding algorithm herself.

The second assumption has held for McEliece's original proposal, but has been proven wrong for many later variants. McEliece [34] suggests a binary Goppa code as the efficiently decodable secret key. The code is disguised through a random column permutation of its generator matrix and left multiplication by a random invertible matrix. Encryption consists of encoding a plaintext message with the public code and adding a random error whose Hamming weight stays within the error-correcting bound of the secret code (which is the same as the bound of the public code). An attacker would then have to correct this error in order to recover the plaintext. For a precise description of the McEliece cryptosystem based on arbitrary efficiently decodable codes, see Section 3 of this paper.

To this day, the McEliece system remains unbroken - if its originally suggested size is adjusted in order to defend against current computer power and incremental speed-ups in decoding algorithms [10]. However, its major disadvantage is the large public key size of the system. For this reason many attempts have been made to find alternatives to McEliece's original proposal using different underlying codes equipped with more compact representations, including both codes with algebraic structure like Reed-Solomon codes and modern codes like low-density parity-check codes with quasi-cyclic structure.

In (almost) all these systems the masking of the secret code consists of a random permutation and rescaling of the columns of a generator matrix along with left multiplication by an invertible matrix. While the left multiplication is clearly just a change of basis of the code, the column operations lead to a so-called monomially equivalent code. Since permuting and rescaling of codeword coordinates leave the Hamming weight invariant, these operations can easily be dealt with when setting up the cryptosystem. On the downside, this type of masking often leaves too much algebraic structure, which an attacker can exploit. Indeed, in most cases the algebraic type of the code remains unchanged: for example, a disguised generalized Reed-Solomon code is a generalized Reed-Solomon code. As a consequence, the knowledge of the code class provides an attacker with further useful information. We give an overview of many proposed variants and attacks in Remark 2, The only codes that appear to resist such an attack are alternant codes [10]. In Section 4 we elaborate on the idea of Baldi et al. [3] by proposing a McEliece cryptosystem where the monomial transformations are replaced by the inverses of (invertible) matrices whose rows have Hamming weight $m=2$. This generalization appears to have the advantage of annihilating the algebraic structure of the secret code without leaving a trace for an attacker to restore it using the distinguisher coming from the Schur square of the public code. Indeed, our simulations show that the Schur square of the public code, a central tool in most attacks, behaves like the Schur square of a random code. This contrasts the system proposed by Baldi et al. [3] for which it has been shown [20] that the dimension of the Schur square is not maximal when the average row weight $m$ is less than $1+R$.

As an alternative to improved masking in algebraic systems, in Section 5 we propose the use of spatially coupled Moderate-Density Parity-Check (SC-MDPC) codes for the McEliece cryptosystem. Low-Density Parity-Check (LDPC) codes were first considered as candidates in [39], but were soon observed to be vulnerable to decoding attacks due to the high density of low-weight codewords in the dual code; a brute force search for such codewords allows attackers to generate a low-density parity-check matrix to efficiently decode the code. Furthermore, general LDPC-based McEliece cryptosystems suffer from large key sizes, motivating quasi-cyclic LDPC (QC-LDPC) codes to be considered [5, 4]. For increased security, Moderate-Density Parity-Check (MDPC) codes were considered in [36], and in particular, quasi-cyclic MDPC (QC-MDPC) codes. Most recently a new attack on all classes of quasi-cyclic MDPC codes has appeared by Guo et al. [24], suggesting 
that the use of quasi-cyclic structure to reduce key size introduces a weakness in security. Since spatially coupled codes are known for their efficient decoding, capacity approaching performance, minimum distance properties, and compact representation [14, 38], the use of these codes offers a promising variant of the cryptosystem. In particular, spatially coupled codes offer a reduced key size without the security cost of rigid quasi-cyclic structure.

In order to check the vulnerability of our proposed systems with respect to brute-force decoding, that is, information-set decoding, we compute the work factor for Stern's algorithm for some sets of parameters and secret generalized Reed-Solomon (GRS) codes in Section 4. Taking the reduced Hamming weight of the errors into account, we show that the system can be made safe with respect to information-set decoding. Though the keys are still large, our variation compares favorably with current post-quantum alternatives. Similarly, in Section 5, we study the work factor for classical decoding attacks and key recover attacks of the spatially coupled MDPC system. In both cases, our variation compares favorably to comparable quasi-cyclic systems.

\section{Coding-Theoretic Preliminaries}

Let us recall some basic notions of coding theory. Throughout let $\mathbb{F}=\mathbb{F}_{q}$ be a finite field with $q$ elements. We endow $\mathbb{F}^{n}$ with the Hamming weight $\mathrm{wt}\left(v_{1}, \ldots, v_{n}\right):=\left|\left\{i \mid v_{i} \neq 0\right\}\right|$. The associated distance is denoted by $\mathrm{d}$, thus $\mathrm{d}(v, w):=\mathrm{wt}(v-w)$. An $[n, k]_{q}$-code $\mathscr{C}$ is a $k$-dimensional subspace of the metric space $\left(\mathbb{F}^{n}, \mathrm{~d}\right)$. The minimum distance of $\mathscr{C}$ is defined as $\mathrm{d}(\mathscr{C})=\min \{\operatorname{wt}(v) \mid v \in \mathscr{C} \backslash\{0\}\}$, and we call $\mathscr{C}$ an $[n, k, d]_{q}$-code (resp. $[n, k, \geq d]_{q}$-code) if it is an $[n, k]_{q}$-code with distance $d$ (resp. at least $d$ ). Any $[n, k]_{q}$-code $\mathscr{C}$ can be written in either of the forms

$$
\mathscr{C}=\operatorname{im}(G):=\left\{u G \mid u \in \mathbb{F}^{k}\right\} \text { and } \mathscr{C}=\operatorname{ker}(H):=\left\{v \in \mathbb{F}^{n} \mid H v^{\top}=0\right\}
$$

for some matrix $G \in \mathbb{F}^{k \times n}$ (whose rows thus form a basis of $\mathscr{C}$ ) and some $H \in \mathbb{F}^{(n-k) \times n}$. We call any such matrix $G$ a generator matrix and $H$ a parity check matrix of $\mathscr{C}$. Define the dual of $\mathscr{C} \subseteq \mathbb{F}^{n}$ as $\mathscr{C}^{\perp}:=\left\{w \in \mathbb{F}^{n} \mid v \cdot w=0\right.$ for all $\left.v \in \mathscr{C}\right\}$, where $w \cdot v$ is the standard dot product. Clearly, if $\mathscr{C}=\operatorname{im}(G)=\operatorname{ker}(H)$ as above, then $\mathscr{C}^{\perp}=\operatorname{im}(H)=\operatorname{ker}(G)$.

Let $\mathscr{C}$ be an $[n, k, \geq d]_{q}$-code. The triangle inequality of the Hamming metric shows that for any $z \in \mathbb{F}^{n}$ satisfying $\mathrm{d}(z, \mathscr{C}):=\min \{\mathrm{d}(z, v) \mid v \in \mathscr{C}\} \leq \frac{d-1}{2}$ there exists a unique codeword $v \in \mathscr{C}$ such that $\mathrm{d}(v, z) \leq \frac{d-1}{2}$. Only codes where this unique codeword can be found efficiently are suitable for error-correcting purposes. There exist various classes of algebraic codes with efficient decoding algorithms, such as generalized Reed-Solomon codes, BCH-codes, alternant codes, Reed-Muller codes, AG-codes. Moreover, graph-based codes are equipped with low-complexity iterative decoders.

Code-based cryptography relies on the fact that decoding up to $(d-1) / 2$ errors with respect to a random linear code is NP-hard; see [9]. Furthermore, the best known decoding algorithms for a random linear code, known as information-set decoding, have time complexity that is exponential in the length and rate.

One such algorithm is due to Stern [48], with a precursor given by Lee/Brickell in [28]. Stern's algorithm aims at finding error vectors with weights $p, p, 0$ when restricted to certain index sets $X, Y, Z$, respectively. Here $X$ and $Y$ are sets of size $k / 2$, and $Z$ has size $\ell$. The parameters $p$ and $\ell$ need to be chosen as to optimize the average run time. A detailed description of the algorithm for codes over arbitrary finite fields can be found in [44, Sec. 3] by Peters. In the same paper, Peters also presents a work factor estimate. For $\mathbb{F}=\mathbb{F}_{q}$ with 
$q=2^{m}$ and the chosen parameters $p$ and $\ell$ this results in

$$
\tilde{W}_{p, \ell}^{\text {bin }}=m S_{p, \ell}\left(\begin{array}{l}
n \\
r
\end{array}\right)\left[\left(\begin{array}{c}
k / 2 \\
p
\end{array}\right)^{2}\left(\begin{array}{c}
n-k-\ell \\
r-2 p
\end{array}\right)\right]^{-1} \text { binary operations, }
$$

where

$$
\begin{gathered}
S_{p, \ell}:=(n-k)^{2}(n+k)+\left[k / 2-p+1+2\left(\begin{array}{c}
k / 2 \\
p
\end{array}\right)(q-1)^{p}\right] \ell \\
+\frac{2 p q(r-2 p+1)(2 q-3)(q-1)^{2 p-2}}{q^{\ell}}\left(\begin{array}{c}
k / 2 \\
p
\end{array}\right)^{2} .
\end{gathered}
$$

The parameters $p$ and $\ell$ have to be determined to minimize $\tilde{W}_{p, \ell}^{\text {bin }}$. The effort can be further reduced by making wise use of computations in a previous round of the iterations. We refer to [10] by Bernstein et al. and [44] by Peters for further details and remark that this re-using of earlier computations is an extension of an original improvement by Canteaut/Chabaud [12]. An additional improvement of Stern's algorithm has been proposed by Finiasz/Sendrier [22]; see also [44, Sec. 6] for arbitrary finite fields. It is interesting to note that in [13], Canto Torres/Sendrier develop a framework which includes all known variants of information set decoding for generic linear codes, and show that all variants of information set decoding that fit within this framework will have asymptotically identical cost when the error rate is sublinear. Thus new information set decoding algorithms that fall within this framework will not significantly change the security of McEliece and its variants.

\section{The McEliece Cryptosystem}

In this section we recall the general set-up for the McEliece cryptosystem and discuss its vulnerability for various choices of underlying codes. The central feature is a code that comes with an efficient decoding algorithm. As before, let $\mathbb{F}=\mathbb{F}_{q}$. We set

$$
\mathscr{G}_{q, n, k, t}=\left\{\begin{array}{l|l}
(\mathscr{C}, \mathscr{D}) \mid \begin{array}{l}
\mathscr{C} \subseteq \mathbb{F}^{n}, \operatorname{dim}(\mathscr{C})=k, \operatorname{dist}(\mathscr{C}) \geq 2 t+1 \\
\mathscr{D} \text { efficient } t \text {-error decoding algorithm for } \mathscr{C}
\end{array}
\end{array}\right\} .
$$

In this terminology, the public key of the cryptosystem is simply the code $\mathscr{C}$, and the decoding algorithm $\mathscr{D}$ is kept secret. Usually, a code is specified by a generator or parity check matrix and knowing an efficient decoding algorithm is equivalent to knowing a particularly structured generator or parity check matrix for the code. In that case the pair $(\mathscr{C}, \mathscr{D})$ above may be replaced with $G$ or $H$, where the latter are such a generator or parity check matrix. The public key will then be a sufficiently general and unstructured generator or parity check matrix of a related code. Such a matrix may be obtained by a sufficient scrambling of the structured matrix. This is the version described below.

Let $\mathrm{GL}_{n}(\mathbb{F}):=\left\{M \in \mathbb{F}^{n \times n} \mid \operatorname{det}(M) \neq 0\right\}$ be the general linear group and define

$$
\mathscr{M}_{n}:=\left\{P \in \mathrm{GL}_{n}(\mathbb{F}) \mid P \text { monomial matrix }\right\},
$$

where a square matrix is called monomial if every row and column contains exactly one nonzero element. In other words, monomial matrices are exactly the products of permutation matrices and nonsingular diagonal matrices. Clearly, $\mathscr{M}_{n}$ is a subgroup of $\mathrm{GL}_{n}(\mathbb{F})$. Monomial matrices can be characterized in the following way. For any $P \in \mathrm{GL}_{n}(\mathbb{F})$ we have

$$
P \text { is monomial } \Longleftrightarrow\left\{\begin{array}{l}
\text { the isomorphism: } \mathbb{F}^{n} \longrightarrow \mathbb{F}^{n}, x \longmapsto x P \\
\text { preserves the Hamming weight. }
\end{array}\right\}
$$


This property plays a central role in code-based cryptosystems because monomial matrices will act on error vectors and therefore do not change the weight of such vectors. This is necessary in the following algorithm so that the legitimate receiver can indeed decode the received cyphertext. We fix a field $\mathbb{F}$ and parameters $k, n$ and assume these data are known to the public.

\section{Construction 1. McEliece Cryptosystem [34]}

(1) Pick a parameter $t$ and a pair $(\mathscr{C}, \mathscr{D}) \in \mathscr{G}_{q, n, k, t}$. Suppose $\mathscr{C}$ is given by the generator matrix $G \in \mathbb{F}^{k \times n}$, which gives rise to the decoding algorithm $\mathscr{D}$ correcting at least $t$ errors.

(2) Pick random matrices $S \in \mathrm{GL}_{k}(\mathbb{F})$ and $P \in \mathscr{M}_{n}$ and set $\bar{G}:=S G P^{-1}$.

(3) Public Key: $(\bar{G}, t)$. Secret Key: $(S, G, P)$.

(4) Plaintext Space: $\mathscr{Z}:=\mathbb{F}^{k}$.

(5) Encryption: Encrypt the plaintext $m \in \mathscr{Z}$ into $c:=m \bar{G}+e$, where $e \in \mathbb{F}^{n}$ is a randomly chosen vector of weight at most $t$.

(6) Decryption:

- Compute $c^{\prime}:=c P=m S G+e P$.

- Decode $c^{\prime}$ with the decoding algorithm $\mathscr{D}$ for $\mathscr{C}$ and denote the output by $m^{\prime}$.

- Return $m^{\prime} S^{-1}$.

Some comments are in order. Step (2) serves to mask the specific generator matrix $G$. Note that left multiplication by $S$ does not change the code generated by $G$, whereas right multiplication by $P$ only mildly changes the code and usually leaves the type of code invariant (such as GRS, alternant, or Reed-Muller). In (5) it is most secure to choose $e$ of weight as large as possible in order to minimize an attacker's success when using information-set decoding. As for (6) we know that $\mathrm{d}\left(c^{\prime}, \operatorname{im} G\right) \leq \mathrm{wt}(e P) \leq t$. Thus the codeword $m S G$ is actually the unique closest codeword to $c^{\prime}$ and therefore applying the decoding algorithm to $c^{\prime}$ will return $m^{\prime}=m S$. Hence $m^{\prime} S^{-1}$ is indeed the plaintext $m$.

Remark 1. A cryptosystem dual to the McEliece system is the Niederreiter system [40]. It is based on a (scrambled) parity check matrix. We refer to [40] or [30. Sec. II.B] for a detailed description. In [30] Li et al. showed that the McEliece system is equivalent to the Niederreiter system [40] if based on the same underlying code. This implies in particular that if one system can be broken then so can the other. However, the Niederreiter system has actually some crucial advantages. Firstly, it allows the public key to be given in systematic form without loss of security, resulting in a reduction of key size and a reduction of work factor for the encryption process. Thus, while for the McEliece system over the field $\mathbb{F}_{2^{m}}$ the public key has size mkn bits, it can be reduced to $m k(n-k)$ bits for the Niederreiter system. Secondly, the McEliece system is actually more vulnerable than the Niederreiter system to a specific type of attack. Indeed, encrypting the same message twice with the McEliece system will most likely result in different error vectors being added, and the difference of the two cyphertexts provides useful information to an attacker who has the chance to use the same message twice. Finally, the Niederreiter system can be used to construct digital signature schemes [15].

Let us now discuss some crucial details concerning the security of the McEliece cryptosystem and its variants. As discussed above, an attacker can either attempt to break the system by using general decoding algorithms, without using any potential special structure of the random-seeming public code, or can attempt to uncover and exploit the structure that leads to an efficient decoding algorithm. The second approach has led to breaks of most proposed variants. We briefly outline the most prominent cases and successful attacks. 


\section{Remark 2.}

(a) Reed-Solomon and Generalized Reed-Solomon (GRS) codes were proposed as a way to reduce key size in the McEliece system by Niederreiter [40]. However, Sidelnikov/Shestakov [47] showed that one can efficiently recover a structured parity-check matrix for the Niederreiter system from the public matrix.

(b) Berger/Loidreau [8] proposed using subcodes of GRS codes, but Wieschebrink [52] extended the Sidelnikov/Shestakov attack to break this system for almost all parameters. This attack is the first one based on Schur products, to be discussed later in this paper. For the same codes, Couvreur at el. [16] derived a nested sequence of subcodes - called a filtration - that expose their vulnerability. Again this attack makes use of the Schur product. In particular, it leads to an attack of GRS based cryptosystems that is different from the one in [47].

(c) Wieschebrink [52] suggested GRS codes with a certain number of random columns inserted. However, using that shortening a GRS code results in a GRS code and comparing the dimensions of certain Schur products, Couvreur et al. [16] could identify the random positions and recover the structure of the secret code.

(d) Sidelnikov [46] proposed binary Reed-Muller codes, but that has been proven insecure by Minder/Shokrollahi [35].

(e) Janwa/Moreno [25] suggested algebraic-geometric codes, but this has been broken by Couvreur/Marquez-Corbella/Pellikaan [17]. The latter derived a t-error-correcting pair with the aid of a filtration that in turn is based on Schur products. The same authors also extended their attack to cryptosystems based on subcodes of AG-codes [18].

(f) Monico et al. [39] proposed LDPC codes for the McEliece system. The code structure was disguised by a sparse invertible matrix acting on the low density parity check matrix. It was pointed out in the original paper that fairly large sizes would be required so that a low density parity check structure cannot be recovered. To avoid this problem medium density parity check (MDPC) codes were proposed by Baldi et. al. [5]. Quasicyclic $(Q C)$ LDPC codes are preferred over general LDPC for their smaller key size, but some classes of QC-LDPC codes and QC-MDPC codes were successfully attacked by Otmani et al. [41] and more recently by Guo et. al. [24]. More general MDPC codes are still promising, as we will discuss in Section 5

(g) Bernstein et al. [11] suggested wild Goppa codes (that is, Goppa codes with Goppa polynomial $\gamma^{q-1}$ for some irreducible polynomial $\gamma$ ) due to their better error-correcting properties. A filtration-type attack based on Schur products has been derived in [19] by Couvreur et al. for the case where the Goppa polynomial comes from a quadratic extension. An additional attack on wild Goppa McEliece was developed in [21] by Faugere et al.

(h) Baldi et al. [3] suggested variants of the McEliece cryptosystem, including adding a rank-1 matrix to the monomial matrix in Step (2). This forces an extra round in the decryption which in essence amounts to testing all values of the underlying field. In [16] Couvreur et al. presented an attack based on Schur products. They determined a specific subspace of the public code with codimension one from which the secret code can be identified with the aid of Wieschebrink's attack [52]. One should note that adding a rank-1 matrix to the monomial matrix leads to a public code that is not monomially equivalent to the secret code anymore, and thus in general is not in the same code class. However, it is exactly this rank-1 property that provides too much structure and thus aids an attacker.

Another idea is to replace the permutation matrix in the monomial transformation with a matrix whose inverse has an average row weight of $m$ where $1<m<<n$. This idea 
probably goes back to Baldi et al. [4] who studied this idea in the context of McEliece versions of LDPC codes. Baldi et al. [3] further elaborated this idea by using values of $m$ in the range $1<m<2$ in the disguising of generalized Reed Solomon codes. For these systems Couvreur et. al. [20] developed a polynomial time attack which works best when the average row weight $m$ is less than $1+R$ where $R$ is the code rate. A similar idea has been employed before in the context of rank-metric codes by Gabidulin et al. [23] but as in the rank-metric coding setting vulnerabilities were discovered by these subcode constructions. See Overbeck [42] for details.

A remaining class of codes that seems to be efficiently decodable and at the same time secure for cryptosystems is subfield-subcodes, such as alternant codes or, more specifically, Goppa codes. The latter are in fact the ones originally suggested by McEliece [34]. The reason is that for these codes the generator matrix $G$ chosen in Step (1) of Construction 1 is itself already highly unstructured. Therefore its scrambled version $\bar{G}$ has - to this day - fended off any attack for recovering the original code. As a consequence, an attacker can only resort to decoding the random looking public code. Choosing sufficiently large parameters $n, k$, any desired security level can be reached; see [10] for latest details on the Goppa code case. However, the disadvantage of this choice is the prohibitively large key size.

Many of the above listed attacks, resulting in an error-correcting pair, amount to computing a nested sequence of subcodes of the public code based on certain Schur products. This strategy relies on the facts that, firstly, the Schur square of GRS and other AG-codes is of much lower dimension than those of a random code, and, secondly, that the attacker knows the algebraic type of the secret code.

We now introduce the Schur product and Schur square and further discuss their importance.

Definition 1. For $x=\left(x_{1}, \ldots, x_{n}\right), y=\left(y_{1}, \ldots, y_{n}\right) \in \mathbb{F}^{n}$ define the star product as $x * y:=$ $\left(x_{1} y_{1}, \ldots, x_{n} y_{n}\right)$. For two codes $\mathscr{C}_{1}, \mathscr{C}_{2} \subseteq \mathbb{F}^{n}$ define the Schur product as

$$
\mathscr{C}_{1} * \mathscr{C}_{2}:=\operatorname{span}_{\mathbb{F}}\left\{x * y \mid x \in \mathscr{C}_{1}, y \in \mathscr{C}_{2}\right\} .
$$

We set $\mathscr{C}^{(2)}:=\mathscr{C} * \mathscr{C}$ and call it the Schur square of $\mathscr{C}$.

Clearly, the star product is commutative and bilinear. Moreover, if the code $\mathscr{C} \subseteq \mathbb{F}^{n}$ has basis $g_{1}, \ldots, g_{k}$, then

$$
\mathscr{C}^{(2)}=\operatorname{span}_{\mathbb{F}}\left\{g_{i} * g_{j} \mid i=1, \ldots, k, j=i, \ldots, k\right\},
$$

and thus, $\operatorname{dim}\left(\mathscr{C}^{(2)}\right) \leq \min \left\{\left(\begin{array}{c}k+1 \\ 2\end{array}\right), n\right\}$. One even has the following generic result.

Proposition 1 ([32, Prop. 2]). Let $n>\left(\begin{array}{c}k+1 \\ 2\end{array}\right)$ and $\mathscr{C}$ be a randomly chosen $[n, k]$-code. Then

$$
\operatorname{Prob}\left(\operatorname{dim}\left(\mathscr{C}^{(2)}\right)=\left(\begin{array}{c}
k+1 \\
2
\end{array}\right)\right)=1 .
$$

However, for a GRS code $\mathscr{C}$ the dimension of $\mathscr{C}^{(2)}$ is actually much smaller. Indeed, a $k$-dimensional GRS code is given by a generator matrix of the form $G=\left(\alpha_{j}^{i} z_{j}\right) \in \mathbb{F}^{k \times n}$ for distinct elements $\alpha_{1}, \ldots, \alpha_{n} \in \mathbb{F}$ and nonzero elements $z_{1}, \ldots, z_{n} \in \mathbb{F}^{*}$. Denoting the resulting code by $\operatorname{GRS}_{n, k}(\alpha, z)$ one easily verifies that

$$
\operatorname{GRS}_{n, k}(\alpha, z) * \operatorname{GRS}_{n, k^{\prime}}\left(\alpha, z^{\prime}\right)=\operatorname{GRS}_{n, k+k^{\prime}-1}\left(\alpha, z * z^{\prime}\right) .
$$


Thus we have

$$
\operatorname{dim}\left(\operatorname{GRS}_{n, k}(\alpha, z)^{(2)}\right)=\min \{2 k-1, n\} .
$$

This almost trivial fact aids an attacker if the cryptosystems are based on GRS codes. In order to see this, one first observes that the Schur square of the secret code and that of the public code are closely related due to the following trivial property of the star product:

$$
\text { for any } x, y \in \mathbb{F}^{n} \text { and } P \in \mathscr{M}_{n} \text { we have }(x P) *(y P)=(x * y) \tilde{P} \text {, }
$$

where $\tilde{P}$ is the monomial matrix $\left(\left(P_{i, j}\right)^{2}\right)$, i.e., each entry of $P$ is squared. As a consequence, using the bilinearity of $*$ we obtain $\operatorname{im}(\bar{G})^{(2)}=\operatorname{im}(G)^{(2)} \widetilde{P^{-1}}$ for the data as in Construction 1 (and where again $\widetilde{P^{-1}}$ is the matrix $P^{-1}$ with each nonzero entry squared). This enables an attacker to make use of the public code in order to compute the Schur square of the underlying secret code - up to the monomial matrix $\tilde{P}$ : by Proposition 1 and Identity (7) she can immediately distinguish the public code from a random code. As mentioned in Remark 2, this is often sufficient to successfully attack the system. In particular, it breaks systems based on GRS codes or AG-codes (the latter for a wide set of parameters).

In the next sections we present two new variations of the McEliece cryptosystem. The first one uses the idea of generalizing the monomial matrix to enhance the masking as described in Remark $2(\mathrm{~h})$ but where we use row weight $m=2$. The other proposes the use of a special class of MDPC codes that have reduced key size and are not quasi-cyclic, thereby avoiding attacks designed for quasi-cyclic codes.

\section{Variation Based on Weight-2 Masking}

Recall the sets (2) and (3). We first propose the following variant of the McEliece cryptosystem. It differs from the classical Construction 1 in that we replace the set $\mathscr{M}_{n}$ of monomial matrices by the set

$$
\mathscr{W}_{n}:=\left\{P \in \mathrm{GL}_{n}(\mathbb{F}) \mid \text { each row of } P \text { has weight } 2\right\} .
$$

Notice that matrices of the form $I_{n}+P$, where $P \in \mathscr{M}_{n}$ are in $\mathscr{W}_{n}$ with high probability. In fact, Sage simulations show that for $q=n=2^{8}$ this probability is at least $96 \%$, whereas for $q=n=2^{9}$ it even goes up to $98 \%$. This allows one to easily create matrices in $\mathscr{W}_{n}$. As we will demonstrate, using matrices from $\mathscr{W}_{n}$ instead of $\mathscr{M}_{n}$ leads to an improved masking of the secret code at the cost of restricting the size of errors to half the original correctable error-size.

Remark 3. We note that the results that follow remain valid if we extend the set $\mathscr{W}_{n}$ to the set of invertible matrices where all rows have weight at most 2 and almost all of them have weight equal to 2.

Construction 2. Variant of McEliece Cryptosystem - Weight-2 Masking

(1) Pick a parameter t and a pair $(\mathscr{C}, \mathscr{D}) \in \mathscr{G}_{q, n, k, t}$ and choose a generator matrix $G$ for $\mathscr{C}$, which gives rise to the decoding algorithm $\mathscr{D}$.

(2) Pick random matrices $S \in \mathrm{GL}_{k}(\mathbb{F})$ and $P \in \mathscr{W}_{n}$ and set $\bar{G}:=S G P^{-1}$.

(3) Public Key: $(\bar{G}, t)$. Secret Key: $(S, G, P)$.

(4) Plaintext Space: $\mathscr{Z}:=\mathbb{F}^{k}$.

(5) Encryption: Encrypt the plaintext $m \in \mathscr{Z}$ into $c:=m \bar{G}+e$, where $e \in \mathbb{F}^{n}$ is a randomly chosen vector such that $\mathrm{wt}(e) \leq t / 2$. 
(6) Decryption:

- Compute $c^{\prime}:=c P=m S G+e P$.

- Decode $c^{\prime}$ using the decoding algorithm $\mathscr{D}$ for $\mathscr{C}$ and denote the output by $m^{\prime}$.

- Return $m^{\prime} S^{-1}$.

Note that, in contrast with the original McEliece system, the matrix $P$ is not monomial, but rather in $\mathscr{W}_{n}$. The definition of this set implies $\operatorname{wt}(e P) \leq 2 \mathrm{wt}(e)$ for any $e \in \mathbb{F}^{n}$, and thus the condition on the error vector $e$ guarantees that $\mathrm{wt}(e P) \leq t$. Therefore, as for Construction 11 decoding of $c^{\prime}=m S G+e P$ yields the correct $m^{\prime}=m S$. In order to make information-set decoding harder one should choose $\mathrm{wt}(e)=t / 2$ or at least close to it.

Remark 4. Just as for the classical system, one can easily formulate a Niederreiter analog for this variant. In this case the secret parity check matrix $H \in \mathbb{F}^{(n-k) \times n}$ is transformed to the public key $\bar{H}:=S^{-1} H P^{\top}$ with $S \in \mathrm{GL}_{n-k}(\mathbb{F})$ and $P \in \mathscr{W}_{n}$ randomly chosen. One may actually choose $S$ such that $\bar{H}$ is in systematic form to reduce the public key size. The plaintext space is $\mathscr{Z}:=\left\{m \in \mathbb{F}^{n} \mid \operatorname{wt}(m) \leq t / 2\right\}$ and the encryption of $m \in \mathscr{Z}$ is given by $c^{\top}:=\bar{H} m^{\top}$. Then one easily checks that decryption works in exactly the same way as for the Niederreiter classical system. We omit further details.

It appears that multiplication by the matrix $P^{-1}$ removes any identifiable algebraic structure from the public code. It changes the code type, since transforming $G$ into $G P^{-1}$ does not amount to a permutation and rescaling of the columns. Note that in general the matrix $P^{-1}$ is not even sparse for $P \in \mathscr{W}_{n}$.

The loss of algebraic structure becomes clear when computing the Schur square $\overline{\mathscr{C}}^{(2)}$ of the public code $\mathscr{\mathscr { C }}=\operatorname{im}(\bar{G})$. The simple identity (8) no longer holds, and the Schur square of the public code appears quite unrelated to the Schur square of the secret code. If we use a GRS code with $2 k-1<n$, this can also be confirmed with the dimension of $\overline{\mathscr{C}}^{(2)}$. Identity (7) tells us that whenever the dimension of $\overline{\mathscr{C}}^{(2)}$ is bigger than $2 k-1$, the public code $\overline{\mathscr{C}}$ is not a GRS code. Similarly, a sufficiently large dimension of the Schur square precludes that the public code is an AG-code (with a certain range of parameters) thanks to [17. Prop. 8]. In fact, the following data suggest that the Schur square of the public code behaves as that of a random code.

Example 1. Sage simulations for the parameters $q=n$ as in Example 3 below and various values of $k$ (with 100 simulations for each chosen set of parameters) show that masking a secret GRS code with a random matrix from $\mathscr{W}_{n}$ results in a Schur square of the public code with the following properties.

(1) The Schur square of $\operatorname{im}(\bar{G})$ attains maximum dimension, i.e., $\min \left\{\left(\begin{array}{c}k+1 \\ 2\end{array}\right), n\right\}$.

(2) The Schur square of randomly taken subspaces of $\mathrm{im}(\bar{G})$ attains maximum dimension.

(3) The Schur square of the orthogonal code $\mathrm{im}(\bar{G})^{\perp}$ attains maximum dimension.

These maximum dimensions occurred in all simulations and thus we conjecture that the above properties appear with probability 1. In other words, with respect to the Schur square, the public code, its subcodes and its dual appear to behave like random codes, see Proposition 1$]$ This is underscored by a recent result by Weger in [51] p.45, Cor. 4] who proved that for fixed $[n, k]$ the probability that the Schur square of $\operatorname{im}(\bar{G})$, where $G$ is a generator matrix of an $[n, k]-G R S$ code, tends to 1 as the field size $q$ goes to infinity. An analogous statement is true for the Niederreiter system [51. p. 49, Cor. 8]. Finally, we wish to add that the masking used in Baldi et al. [3] using transformations having average row weight $m$ with $1<m<2$ transforms a GRS code into a code whose Schur square does not have full rank, see [20]. 
Example 2. Let $\mathscr{H}_{q}$ be the Hermitian curve with affine model given by

$$
\mathscr{H}_{q}: \quad x^{q}+x=y^{q+1} .
$$

The projective curve $\mathscr{H}_{q}$ has a single point at infinity, is smooth of genus $g=\frac{q(q-1)}{2}$ and has $q^{3}+1$ points over $\mathbb{F}_{q^{2}}$. This is the largest number of points possible over $\mathbb{F}_{q^{2}}$ for a curve

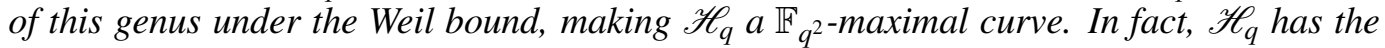
largest genus possible for a $\mathbb{F}_{q^{2}}$-maximal curve. AG-codes on Hermitian curves have been widely studied [31] 49] because these codes may have length up to $n=q^{3}$ with alphabet $\mathbb{F} q^{2}$.

Let $\mathscr{H}_{q}\left(\mathbb{F}_{q^{2}}\right)$ be the set of points on $\mathscr{H}_{q}$ defined over $\mathbb{F}_{q^{2}}$, and let $P_{\infty}$ denote the unique point at $\infty$. Let $\mathscr{P}=\left(P_{1}, P_{2}, \ldots, P_{n}\right)$, where $P_{i} \in \mathscr{H}_{q}\left(\mathbb{F}_{q^{2}}\right) \backslash\left\{P_{\infty}\right\}$ for $1 \leq i \leq q^{3}$. Let $E$ be the divisor $a P_{\infty}$, where $a$ is any natural number with $2 g-2<a<q^{3}$. The one-point $A G$ code $C_{a}=C_{L}\left(\mathscr{H}_{q}, \mathscr{P}, E\right)$ (see [49] Section 8.3] and [17. 18]) is a code of length $n=q^{3}$, dimension $k=a+1-g$, and minimum distance $d \geq q^{3}-a$ [49] Proposition 8.3.3].

The Schur square of the code $C_{a}$ is exactly the code $C_{2 a}$, which has dimension $k^{*}=$ $\min \{n, 2 a+1-g\}$. Thus if $2 a+1-g<n$, a Hermitian code $C_{a}$ can be distinguished from a random code of equal dimension using the Schur square.

Sage simulations were carried out on masked Hermitian codes for the following parameter sets:

- The curve $\mathscr{H}_{4}$, with $a=20, a=25$, and $a=30$, where the code $C_{a}$ is defined over $\mathbb{F}_{16}$, with length $n=64$ in all cases. The code $C_{a}$ has dimension $k=15, k=20$, and $k=25$ respectively.

- The curve $\mathscr{H}_{5}$, with $a=20, a=30$, and $a=40$, where the code $C_{a}$ is defined over $\mathbb{F}_{25}$, with length $n=125$ in all cases. The code $C_{a}$ has dimension $k=11, k=21$, and $k=31$ respectively.

In each case, the code was masked with a random matrix from $\mathscr{W}_{n}$ and the dimension of the Schur square of the masked code was computed in 100 trials. In all cases, the Schur square of $\operatorname{im}(\bar{G})$ attains maximum dimension, i.e., $\min \left\{\left(\begin{array}{c}k+1 \\ 2\end{array}\right), n\right\}$. Thus the algebraic structure appears to be well-hidden for Hermitian codes as well.

Summarizing, it appears that the vulnerability of our variants depends mainly on the success of direct decoding attacks. We close this section with the following work factor estimates.

Example 3. We consider the proposed variant of the McEliece cryptosystem and the Niederreiter dual (see Remark 4) based on GRS codes with parameters $[q, n, k]$ as in the table below. The public key size is given in bytes and we estimate the work factor for Stern decoding with the aid of (1), and where $r$ is the number of errors to be corrected (the work factor for the Lee-Brickell algorithm [28] is higher in each case and thus omitted). For an [n,k]-GRS code we have $t=(n-k) / 2$ and thus we may add $r=t / 2$ errors in the encryption. The estimate for $\tilde{W}_{p, \ell}^{\text {bin }}$ is the minimum over all possible values for $p$ and $\ell$.

\begin{tabular}{|c|c|c|c|}
\hline$[q, n, k]$ & key size McEliece & key size Niederreiter & $\tilde{W}_{p, \ell}^{\text {bin }}$ for $r=\lfloor t / 2\rfloor$ \\
\hline \hline$\left[2^{8}, 2^{8}, 2^{7}\right]$ & 32,768 & 16,384 & $2^{53}$ \\
\hline$\left[2^{9}, 450,230\right]$ & 116,437 & 56,925 & $2^{81}$ \\
\hline$\left[2^{9}, 470,240\right]$ & 126,900 & 62,100 & $2^{83}$ \\
\hline$\left[2^{9}, 2^{9}, 200\right]$ & 115,200 & 70,200 & $2^{82}$ \\
\hline$\left[2^{9}, 2^{9}, 250\right]$ & 144,000 & 73,687 & $2^{88}$ \\
\hline
\end{tabular}


Let us put these numbers into context. After an optimized attack on the classical McEliece system based on binary Goppa codes, Bernstein et al. [10] recommended a binary Goppa code of size at least $[n, k]=[1632,1269]$ (based on a Goppa polynomial in $\mathbb{F}_{2^{11}}[x]$ of degree $t=33$ ). This leads to a key size of 57,580 bytes if used with the Niederreiter system. Only this size guarantees an 80-bit security (i.e., a work factor for their attack with at least $2^{80}$ binary operations), see [10]. Note that RSA-1024, having 80-bit security, has a key size of 256 bytes. It seems likely that a similar optimization of Stern's algorithm plus additional software speedups as in [10] reduces the according work factors in above table. More recently, further improvements of Stern's algorithm have been presented by Becker et al. [7] and May et al. [33], but as in [10] they are tailored to binary codes. It is left to future research whether their results can be generalized to our case in order to lower the work factor even further. Notwithstanding further reductions, it appears that our proposed system compares quite well to the binary $[1632,1269]$-Goppa code.

\section{A Variation Based on Spatially Coupled MDPC Codes}

In this section, we examine the use of a class of spatially coupled moderate density paritycheck (SC-MDPC) codes for the McEliece cryptosystem. Spatially coupled codes are known for their capacity-approaching performance and improved decoding thresholds (i.e. lowest channel quality where error correction is possible) [27]. As noted in Remark 2, low density codes and some quasi-cyclic codes are vulnerable to attack. While the decoding performance of general MDPC codes decreases as density increases, spatially coupled codes typically have the opposite effect [27]. We note further that spatially coupled codes may be obtained using several methods (e.g., [45], [2]). Our focus will be on the construction of SC-MDPC codes using MDPC convolutional codes, specifically terminated convolutional codes arising from quasi-cyclic MDPC codes. For background on terminated convolutional codes arising from quasi-cyclic codes, we refer the reader to [29, 50]. The resulting SCMDPC code may be represented using a bipartite Tanner graph [26] and decoded using standard message-passing belief propagation algorithms [43, 26]. This construction approach ensures that the resulting codes are not quasi-cyclic and yet still yield a smaller key size than general MDPC codes.

Throughout this section let $\mathbb{F}=\mathbb{F}_{2}$. We will use $\mathbb{F}[D]^{r \times n}$ and $\mathbb{F}(D)^{r \times n}$ to denote the space of $r \times n$ matrices with entries in $\mathbb{F}[D]$ and $\mathbb{F}(D)$, respectively. As before, $\mathrm{GL}_{r}(\mathbb{F})$ denotes the group of $r \times r$ invertible matrices with entries in $\mathbb{F}$. For a polynomial $f=$ $\sum_{i=0}^{n} f_{i} D^{i} \in \mathbb{F}[D]$ we define the weight of $f$ as number of nonzero coefficients, thus wt $(f)=$ $\left|\left\{i \mid f_{i} \neq 0\right\}\right|$. Note that $\mathrm{wt}(f+g) \leq \mathrm{wt}(f)+\mathrm{wt}(g)$ and $\operatorname{wt}(f g) \leq \mathrm{wt}(f) \mathrm{wt}(g)$ for all polynomials $f, g$. Furthermore, we recall the notion of a circulant matrix. For a vector $a=\left(a_{1}, \ldots, a_{m}\right) \in \mathbb{F}^{m}$ denote by $C_{a} \in \mathbb{F}^{m \times m}$ the circulant matrix with $i$-th row given by the cyclic shift $\left(a_{i}, \ldots, a_{m}, a_{1}, \ldots, a_{i-1}\right)$ for $i=1, \ldots, m$. Moreover, we associate with $a$ and $C_{a}$ the polynomial $f_{a}=\sum_{i=0}^{m-1} a_{i+1} D^{i} \in \mathbb{F}[D]$.

Let $H \in \mathbb{F}^{r m \times m n}$ be the parity check matrix of a QC-MDPC code, that is, $H=\left(C_{a_{i j}}\right)_{\substack{i=1, \ldots, r \\ j=1, \ldots, n}}$, where $C_{a_{i j}} \in \mathbb{F}^{m \times m}$ are circulant matrices. We associate with it the polynomial matrix $H(D)=\left(f_{i j}\right) \in \mathbb{F}[D]^{r \times n}$, where $f_{i j}:=f_{a_{i j}}$ is the polynomial associated with the circulant $C_{a_{i j}}$. Then $H(D)$ is a polynomial parity-check representation of a SC-MDPC code. It will represent the private key in the McEliece cryptosystem. Let $p, r$ be parameters such that $\mathrm{wt}\left(f_{i j}\right) \leq p$ and $\operatorname{deg}\left(f_{i j}\right) \leq d$ for all $i, j$.

Let now $H^{\prime}(D)=T H(D)$ for some $T \in \mathrm{GL}_{r}(\mathbb{F})$. Then clearly, $H^{\prime}(D)$ and $H(D)$ have the same row space. (It is assumed here that $H(D)$ and $H^{\prime}(D)$ have rank $r$. This is guaranteed with high probability when the choice of the entries in $H(D)$ are sufficiently random.) From 
the properties of the weight of polynomials it follows that $\mathrm{wt}(f) \leq r p$ and $\operatorname{deg}(f) \leq d$ for all entries $f$ of $H^{\prime}(D)$.

Remark 5. More generally, one can use a non-singular matrix $T(D) \in \mathbb{F}[D]^{r \times r}$ having at most $t$ non-zero coefficients in each entry to make it harder for an eavesdropper to guess the structure of $H(D)$. However, the number of non-zero coefficients in each polynomial entry of $H^{\prime}(D)$ will be larger as a result, increasing the overall key size.

We now compute a specific generator matrix for $H^{\prime}(D)$ in order to give an upper bound for the key size of our public key. Recall that $H(D)$ has rank $r$. Thus, without loss of generality we may assume $H^{\prime}(D)=[C(D) \mid M(D)]$, where $C(D) \in \mathbb{F}[D]^{r \times r}$ is nonsingular and $M(D)$ denotes the last $n-r$ columns of $H^{\prime}(D)$. Then the matrix $H^{\prime \prime}(D):=$ $C(D)^{-1} H^{\prime}(D)$ is of the form $H^{\prime \prime}(D)=[I \mid P(D)]$, where $P(D)=\frac{1}{\operatorname{det}(C(D))} \operatorname{Adj}(C(D)) M(D) \in$ $\mathbb{F}(D)^{r \times(n-r)}$ and where $\operatorname{Adj}(C(D))$ is the classical adjoint of the matrix $C(D)$. Define $G^{\prime}(D):=\left[P^{\prime}(D)^{T} \mid \operatorname{det}(C(D)) \cdot I_{k}\right]$, where $k:=n-r$ and $P^{\prime}(D)=\operatorname{Adj}(C(D)) M(D)$. Then clearly $G^{\prime}(D) H^{\prime}(D)^{T}=0$ and thus $G^{\prime}(D)$ is a polynomial generator matrix for the code with parity-check matrix $H^{\prime}(D)$. Furthermore, it is easy to see that every entry $f$ in $G^{\prime}(D)$ satisfies $\operatorname{wt}(f) \leq r !(r p)^{r}$ and $\operatorname{deg}(f) \leq d^{r}$.

We will use $G^{\prime}(D)$ for the public key. The number of distinct non-zero polynomial entries of $G^{\prime}(D)$ is $r(n-r)+1$. Since the degree of each entry is at most $d^{r}$, each nonzero coefficient requires $\log _{2}\left(d^{r}\right)$ bits to represent it, and thus the key size is $(r(n-r)+$ 1) $r !(r p)^{r} r \log _{2}(d)$ bits.

Let $d^{\prime}$ be the actual maximum degree among all entries in $G^{\prime}(D)$. A message to be encrypted is represented in the form

$$
m(D)=\left(m_{1}(D), m_{2}(D), \ldots, m_{k}(D)\right) \in \mathbb{F}[D]^{k},
$$

where $\operatorname{deg}\left(m_{i}(D)\right) \leq z-1-d^{\prime}$ for $i=1, \ldots, k$ for some chosen $z$. The message $m(D)$ is encoded as

$$
c(D)=m(D) G^{\prime}(D)+e(D),
$$

where $e(D)=\left(e_{1}(D) \ldots, e_{n}(D)\right) \in \mathbb{F}[D]^{n}$ is an error polynomial with $\operatorname{deg}\left(e_{i}(D)\right) \leq z-1$ for all $i$ and $\sum_{i=1}^{n} \mathrm{wt}\left(e_{i}(D)\right)=t$. The resulting SC-MDPC code has dimension approximately $k\left(z-d^{\prime}-1\right)$, block length $n z$, and rate approximately $\frac{k\left(z-d^{\prime}-1\right)}{n z}$.

The intended receiver takes an encrypted message $c(D)$ and uses the graphical representation of $H(D)$ (i.e., the private key) and the belief propagation algorithm to decode $c(D)$. The value of $t$ in the choice of the error vector $e(D)$ is such that $t$ is close to the maximum value for the belief propagation decoder on $H(D)$ to recover the message $m(D)$ with high probability (e.g, probability $1-10^{-6}$ ).

Remark 6. Alternatively, one can use $G^{\prime \prime}(D)=S G^{\prime}(D)$ as the public key, where $S \in$ $\mathrm{GL}_{n-r}(\mathbb{F})$ is a random matrix and is known as part of the private key. This will increase the security of the system at the cost of an increase in key size.

We now summarize this variation of the McEliece cryptosystem.

Denote by $\mathscr{H}_{n, m, r, z, d, t}$ the set of all pairs $(\mathscr{C}, \mathscr{T})$, where $\mathscr{C} \subseteq \mathbb{F}^{m n}$ is a QC-MDPC code with circulant block size $m$ and associated convolutional SC-MDPC code of dimension $k:=n-r$ over $\mathbb{F}(D)$ and where $\mathscr{T}$ represents a Tanner graph of that code that can correct up to $t$ errors using a message passing decoder with memory (edge-spreading depth) $d$. Here, $z$ determines the length of the terminated SC-MDPC code.

Construction 3. Variant of McEliece Cryptosystem - SC-MDPC 
(1) Pick a parameter t and a pair $(\mathscr{C}, \mathscr{T}) \in \mathscr{H}_{n, m, r, z, d, t}$. Suppose $\mathscr{C}$ corresponds to paritycheck matrix $H(D) \in \mathbb{F}[D]^{r \times n}$, which gives rise to the Tanner graph $\mathscr{T}$.

(2) Pick a random $T \in \mathrm{GL}_{r}(\mathbb{F})$, and form $H^{\prime}(D)=T H(D)$. Let $G^{\prime}(D)$ be a corresponding generator matrix as described earlier with maximum degree $d^{\prime}$ among its entries.

(3) Public Key: $\left(G^{\prime}(D), t, z, d^{\prime}\right)$.

Secret Key: $(T, H(D))$.

(4) Plaintext Space: $\left\{\left(m_{1}(D), m_{2}(D), \ldots, m_{k}(D)\right) \in \mathbb{F}[D]^{k} \mid \operatorname{deg}\left(m_{i}(D)\right) \leq z-1-d^{\prime}\right\}$.

(5) Encryption: Encrypt the plaintext $m(D)$ into $c(D):=m(D) G^{\prime}(D)+e(D)$, where $e(D) \in$ $\mathbb{F}_{2}[D]^{n}$ is randomly chosen such that $\operatorname{deg}\left(e_{i}(D)\right) \leq z-1$ for all $i=1, \ldots, n$ and $\sum_{i=1}^{n} \operatorname{wt}\left(e_{i}(D)\right) \leq$ $t$.

(6) Decryption: Decode $c(D)$ using a belief propagation algorithm on the graphical representation of $H(D)$. Return the resulting output $m(D)$.

We now assess the security of the SC-MDPC cryptosystem.

Classical decoding attacks: Because the published generator matrix $G^{\prime}(D)$ is denser than $H(D)$ and hides the underlying structure of the spatially coupled structure of the code $\mathscr{C}$, this system is not specifically compromised with respect to classical decoding attacks like the information-set decoding described in [48]. Furthermore, the proposed system is better than systems utilizing quasi-cyclic codes, since the cyclic shift property of codewords may be exploited to diminish the complexity of attacks on the QC-MDPC based cryptosystem [6]. As the SC-MDPC codes proposed here are no longer QC due to termination, classical decoding attacks on the SC-MDPC based cryptosystem have a work factor in the order of $W\left(n z, k\left(z-d^{\prime}-1\right), t\right)$, where $W(N, K, w)$ represents the number of elementary operations needed to successfully search for codewords of weight $w$ in a code with length $N$ and dimension $K$ [7, 6]. Thus, SC-MDPC based cryptosystems are expected to be better than QC-MDPC based cryptosystems against classical decoding attacks.

Key recovery attacks (KRA): These involve recovering the private key from the public key and using the private key to decrypt the ciphertext. Even when the private key is not exactly recovered, the attack may be successful if an alternate private key is found that can successfully decode the ciphertext. In the proposed SC-MDPC cryptosystem, an attacker can reconstuct $H^{\prime \prime}(D)$ from the public key $G^{\prime}(D)$. $H^{\prime \prime}(D)$ has row weight upper bounded by $(n-r+1) r !(r p)^{r}$. As $H^{\prime \prime}(D)$ is expected to have inferior error correction performance compared to the matrix $H(D)$ with message passing decoding, one approach is to transform $H^{\prime \prime}(D)$ into a sparse matrix using matrix transformations. An alternative approach for the KRA involves guessing the rows of the dual code using the public key $G^{\prime}(D)$. From [6, 37], it can be shown that if the row weight of $H(D)$ is $p n$, then the work factor for KRA attack on the SC-MDPC system is in the order of $W\left(n z, r z-(n-r)\left(d^{\prime}+1\right), n p\right)$.

Example 4. Let $H(D)$ be the $2 \times 5$ polynomial matrix

$$
H(D)=\left(\begin{array}{ccccc}
1+D+D^{25}+D^{31}+D^{50} & D^{3}+D^{30} & D^{3}+D^{17}+D^{29} & D+D^{32}+D^{48} & 1+D^{4}+D^{39}+D^{50} \\
D+D^{2}+D^{30} & D^{3}+D^{27}+D^{38} & D^{2} & D^{2}+D^{23}+D^{37} & 1+D+D^{44}
\end{array}\right) .
$$

Then $\operatorname{wt}(f) \leq p:=5$ and $\operatorname{deg}(f) \leq d:=50$ for all entries $f$ of $H(D)$. Choosing $T=\left(\begin{array}{ll}1 & 1 \\ 0 & 1\end{array}\right)$ and setting $H^{\prime}(D):=T H(D)$, we obtain the associated generator matrix

$$
G^{\prime}(D)=\left(\begin{array}{ccccc}
a_{1}(D) & a_{2}(D) & \Delta(D) & 0 & 0 \\
a_{3}(D) & a_{4}(D) & 0 & \Delta(D) & 0 \\
a_{5}(D) & a_{6}(D) & 0 & 0 & \Delta(D)
\end{array}\right)
$$

where 


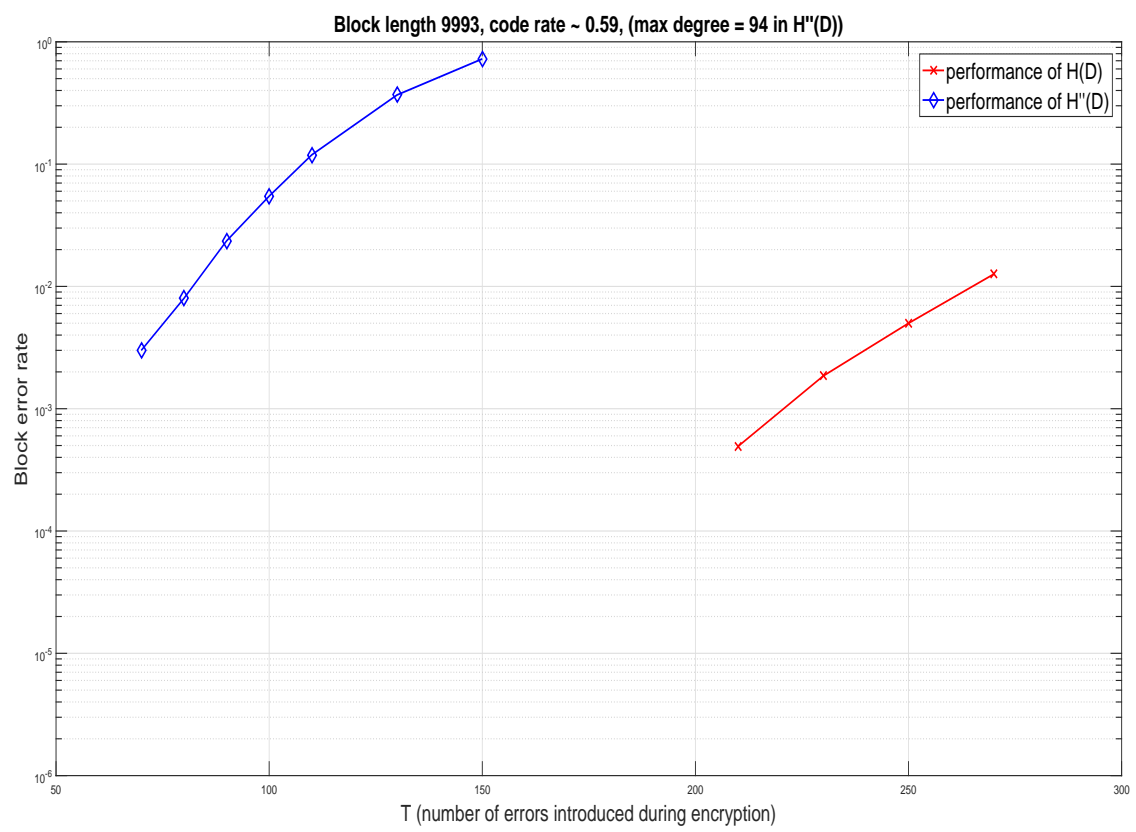

Figure 1: Performance of the SC MDPC cryptosystem for both the intended receiver using $H(D)$, and an attacker using $H^{\prime \prime}(D)$.

$$
\begin{aligned}
& \Delta(D)=D^{3}+D^{5}+D^{27}+D^{31}+D^{32}+D^{33}+D^{34}+D^{38}+D^{39}+D^{52}+D^{53}+D^{58}+D^{60}+D^{63}+D^{69}+D^{77}+D^{88}, \\
& a_{1}(D)=D^{5}+D^{6}+D^{20}+D^{30}+D^{41}+D^{44}+D^{55}+D^{56}+D^{67}, \\
& a_{2}(D)=D^{2}+D^{3}+D^{4}+D^{5}+D^{18}+D^{19}+D^{27}+D^{30}+D^{31}+D^{47}+D^{52}+D^{59}, \\
& a_{3}(D)=D^{4}+D^{5}+D^{26}+D^{28}+D^{32}+D^{35}+D^{39}+D^{40}+D^{51}+D^{53}+D^{59}+D^{67}+D^{70}+D^{75}+D^{86}, \\
& a_{4}(D)=D^{23}+D^{24}+D^{27}+D^{31}+D^{34}+D^{37}+D^{38}+D^{48}+D^{49}+D^{50}+D^{52}+D^{54}+D^{68}+D^{73}+D^{78}+D^{87}, \\
& a_{5}(D)=D^{4}+D^{7}+D^{27}+D^{30}+D^{38}+D^{47}+D^{53}+D^{66}+D^{74}+D^{88}, \\
& a_{6}(D)=1+D+D^{5}+D^{6}+D^{25}+D^{26}+D^{30}+D^{31}+D^{32}+D^{34}+D^{40}+D^{41}+D^{44}+D^{45}+D^{50}+D^{52}+D^{75}+D^{80}+D^{94} .
\end{aligned}
$$

Thus the entries of $G^{\prime}(D)$ have maximal degree $d^{\prime}=94$ (as opposed to the upper bound $d^{r}=50^{2}$ ) and the sum of the weights of all entries is 98. Hence the key size is around $98 \cdot \log _{2}(94)=643$ (as opposed to the upper bound $(r(n-r)+1) r !(r p)^{r} \log _{2}\left(d^{r}\right)$ ). The final SC-MDPC code with generator matrix $G^{\prime}(D)$ obtained from the above construction uses $z=2000$. The overall block length is $N=5(2000)=10000$ bits and dimension $K \sim$ $(n-r) z-(n-r)\left(d^{\prime}+1\right)=5715$. Using simulations, the value $t=140$ was found to be a good choice for the SC-MDPC cryptosystem with these parameters. Decoding via $H(D)$ using message passing decoder is able to correct errors of weight $t$. Using the analysis in [6], we can show that for classical decoding attacks, the cryptosystem in Example 5.5 achieves $>2^{80}$ level of security for $t \sim 140$. However, for key recovery attacks, the minimum row weight of the matrix $H(D)$ in Example 4 is too small to guarantee the same security. Denser parity check matrices (see Table 2 in [36]) are necessary to achieve a high level of security against KRA and will result in a larger key size.

Figure $\square$ shows the performance of the SC-MDPC cryptosystem designed from Example 4 The choice of entries in $H(D)$ resulted in some isolated vertices in the Tanner graph of the SC-MDPC code that were discarded. Thus, the overall blocklength is slightly less than the intended block length of 10,000 bits, and the code rate is approximately 0.59. The figure shows that the intended receiver can decode with low probability of error using a belief propagation decoder when the number of injected errors during encryption is $t \leq 140$. 
An attacker who can construct $H^{\prime \prime}(D)$ has probability $\sim 1$ of failing to decode when the number of bit errors injected during encryption is around 140 errors. Although no error floor was observed in the simulation, error floor performance at very low block failure rates of these codes with message passing decoding needs to be investigated further. This will not change the attacker's ability but could make the system less robust for the receiver.

Acknowledgements We would like to thank the organizers of the IPAM workshop on Algebraic Geometry for Coding Theory and Cryptography for inviting us to the event. Thanks also go to Mike O'Sullivan for helpful conversations and to the anonymous referee for kind suggestions. HGL was partially supported by the National Science Foundation Grant DMS-1210061 and by the grant \#422479 from the Simons Foundation. JB was supported by the US Department of Education GAANN Grant P200A120068. JR was partially supported by the Swiss National Science Foundation under grant no. 169510. BM was partially supported by the National Security Agency under grant H98230-16-1-0300.

\section{References}

[1] Report on Post-Quantum Cryptography. Technical report, National Institute of Standards and Technology, February 2016. NISTIR 8105.

[2] B. Amiri, A. Reisizadehmobarakeh, H. Esfahanizadeh, J. Kliewer, and L. Dolecek. Optimized design of finite-length separable circulant-based spatiallycoupled codes: An absorbing set-based analysis. IEEE Trans. on Communications, 6778(c):1-1, 2016.

[3] M. Baldi, M. Bianchi, F. Chiaraluce, J. Rosenthal, and D. Schipane. Enhanced public key security for the McEliece cryptosystem. J. Cryptol, 29:1-27, 2016. arXiv: 1108.2462, 2011.

[4] M. Baldi, M. Bodrato, and F. Chiaraluce. A new analysis of the McEliece cryptosystem based on QC-LDPC codes. In Proc. Security and Cryptography for Networks 2008 (Amalfi, Italy), LNCS vol. 5229, page 246262, 2008.

[5] M. Baldi and F. Chiaraluce. Cryptanalysis of a new instance of mceliece cryptosystem based on qc-ldpc codes. In 2007 IEEE International Symposium on Information Theory, pages 2591-2595. IEEE, 2007.

[6] M. Baldi, P. Santini, and F. Chiaraluce. Soft McEliece: MDPC code-based McEliece cryptosystems with very compact keys through real-valued intentional errors. arXiv preprint arXiv:1606.01040, 2016.

[7] A. Becker, A. Joux, A. May, and A. Meurer. Decoding random binary linear codes in $2^{n / 20}$ : How $1+1=0$ improves information set decoding. In Eurocrypt 2012, LNCS vol. 7237, pages 520-536, 2012.

[8] T. Berger and P. Loidreau. How to mask the structure of codes for cryptographic use. Des. Codes Cryptogr., 35:63-79, 2005.

[9] E. Berlekamp, R. McEliece, and H. van Tilborg. On the inherent intractability of certain coding problems. IEEE Trans. Inform. Theory, IT-24:384-386, 1978.

[10] D. Bernstein, T. Lange, and C. Peters. Attacking and defending the McEliece cryptosystem. Post-Quantum Cryptography, pages 31-46, 2008.

[11] D. Bernstein, T. Lange, and C. Peters. Wild McEliece. In Selected Areas in Cryptography, pages 143-158, 2010. 
[12] A. Canteaut and F. Chabaud. A new algorithm for finding minimum-weight words in a linear code: application to McEliece's cryptosystem and to narrowsense BCH codes of length 511. IEEE Trans. Inform. Theory, IT-44:367-378, 1998.

[13] R. Canto Torres and N. Sendrier. Analysis of information set decoding for a sub-linear error weight. In Takagi, editor, Post-Quantum Cryptography, LNCS vol. 9606, pages 144-161. Springer Cham, 2016.

[14] D. J. Costello, Jr., L. Dolecek, T. E. Fuja, J. , Kliewer, D. J. M. Mitchell, and R. Smarandache. Spatially coupled sparse codes on graphs: Theory and practice. IEEE Communications Magazine, 52(7):168-176, 2014.

[15] N. Courtois, M. Finiasz, and N. Sendrier. How to achieve a McEliece-based digital signature scheme. In ASIACRYPT 2001, LNCS vol. 2248, pages 157-174. Springer, 2001.

[16] A. Couvreur, P. Gaborit, V. Gauthier-Umaña, A. Otmani, and J.-P. Tillich. Distinguisher-based attacks on public-key cryptosystems using Reed-Solomon codes. Des. Codes Cryptogr., 73:641-666, 2014.

[17] A. Couvreur, I. Márquez-Corbella, and R. Pellikaan. A polynomial time attack against algebraic geometry code based public key cryptosystems. In 2014 IEEE International Symposium on Information Theory, pages 1446-1450, 2014.

[18] A. Couvreur, I. Márquez-Corbella, and R. Pellikaan. Cryptanalysis of publickey cryptosystems that use subcodes of algebraic geometry codes. In Coding Theory and Applications, CIM Series in Mathematical Sciences 3 (R. Pinto, P. Rocha Malonek, P. Vettori, eds.), pages 133-140. Springer, 2015.

[19] A. Couvreur, A. Otmani, and J.-P. Tillich. Polynomial time attack on wild McEliece over quadratic extensions. In Advances in Cryptology Eurocrypt 2014, LNCS vol. 8441, pages 17-39. Springer, 2014.

[20] A. Couvreur, A. Otmani, J.-P. Tillich, and V. Gauthier-Umaña. A polynomialtime attack on the BBCRS scheme. In Public-key cryptography-PKC 2015, volume 9020 of Lecture Notes in Comput. Sci., pages 175-193. Springer, Heidelberg, 2015.

[21] J.-C. Faugère, L. Perret, and F. de Portzamparc. Algebraic attack against variants of McEliece with Goppa polynomial of a special form. In Sarkar and Iwata, editors, Advances in Cryptology. ASIACRYPT 2014. LNCS, vol. 8873, pages 21-41. Springer Berlin Heidelberg, 2014.

[22] M. Finiasz and N. Sendrier. Security bounds for the design of code-based cryptosystems. In Advances in cryptology ASIACRYPT 2009, LNCS vol. 5912, pages 88-105. Springer, 2009.

[23] E. Gabidulin, A. Paramonov, and O. Tretjakov. Ideals over a non-commutative ring and their application in cryptology. In D. Davies, editor, Advances in Cryptology, EUROCRYPT'91, volume 547 of Lecture Notes in Computer Science, pages 482-489. Springer Berlin Heidelberg, 1991.

[24] Q. Guo, T. Johansson, and P. Stankovski. A key recovery attack on MDPC with CCA security using decoding errors. Cryptology ePrint Archive, Report 2016/858, 2016.

[25] H. Janwa and O. Moreno. McEliece public key cryptosystems using algebraicgeometric codes. Des. Codes Cryptogr., 8:293-307, 1996. 
[26] F. Kschischang, B. Frey, and H.-A. Loeligar. Factor graphs and the sum-product algorithm. IEEE Trans. Inform. Theory, 47(2):498-519, 2001.

[27] S. Kudekar, T. J. Richardson, and R. L. Urbanke. Threshold saturation via spatial coupling: why convolutional LDPC ensembles perform so well over the BEC. IEEE Trans. Inform. Theory, 57(2):803-834, 2011.

[28] P. Lee and E. Brickell. An observation on the security of McEliece's public key cryptosystem. In Advances in Cryptology Eurocrypt 1988, LNCS vol. 330, pages 275-280. Springer, 1989.

[29] Y. Levy and D. J. Costello Jr. An algebraic approach to constructing convolutional codes from quasi-cyclic codes. DIMACS Series in Discrete Mathematics and Theoretical Computer Science, 14:189-198, 1993.

[30] Y. X. Li, R. H. Deng, and X. M. Wang. On the equivalence of McEliece's and niederreiter's public-key cryptosystems. IEEE Trans. Inform. Theory, IT40:271-273, 1994.

[31] J. Little, K. Saints, and C. Heegard. On the structure of Hermitian codes. Journal of Pure and Applied Algebra, 121:293-314, 1997.

[32] I. Màrquez-Corbella and R. Pellikaan. Error-correcting pairs for a public-key cryptosystem. arXiv: 1205.3647, 2012.

[33] A. May, A. Meurer, and E. Thomae. Decoding random linear codes in $\tilde{\mathscr{O}}\left(2^{0.054 n}\right)$. In ASIACRYPT 2011, LNCS vol. 7073, pages 107-124, 2011.

[34] R. McEliece. A public-key cryptosystem based on algebraic coding theory. In DSN Progress Report, volume 42, pages 114-116, 1978.

[35] L. Minder and A. Shokrollahi. Cryptanalysis of the Sidelnikov cryptosystem. In Advances in Cryptology Eurocrypt 2007, LNCS vol. 4515, pages 347-360. Springer, 2007.

[36] R. Misoczki, J.-P. Tillich, N. Sendrier, and P. Baretto. MDPC-McEliece: New McEliece variants from moderate density parity-check codes. In 2013 IEEE International Symposium on Information Theory, pages 2069-2073, 2013.

[37] R. Misoczki, J.-P. Tillich, N. Sendrier, and P. S. Barreto. MDPC-McEliece: New McEliece variants from moderate density parity-check codes. In Information Theory Proceedings (ISIT), 2013 IEEE International Symposium on, pages 20692073. IEEE, 2013.

[38] D. J. M. Mitchell, M. Lentmaier, and D. J. Costello, Jr. Spatially coupled LDPC codes constructed from protographs. IEEE Trans. Inform. Theory, 61(9):48664889, 2015.

[39] C. Monico, J. Rosenthal, and A. Shokrollahi. Using low density parity check codes in the McEliece cryptosystem. In Proceedings of the 2000 IEEE International Symposium on Information Theory, page 215, Sorrento, Italy, 2000.

[40] H. Niederreiter. Knapsack-type cryptosystems and algebraic coding theory. Problems of Control and Information Theory, 15:159-166, 1986.

[41] A. Otmani, J. Tillich, and L. Dallot. Cryptanalysis of two McEliece cryptosystems based on quasi-cyclic codes. In Proc. First International Conference on Symbolic Computation and Cryptography (SCC 2008), Beijing, China, 2008.

[42] R. Overbeck. Structural attacks for public key cryptosystems based on Gabidulin codes. J. Cryptology, 21(2):280-301, 2008. 
[43] J. Pearl. Probabilistic reasoning in intelligent systems: Networks of plausible inference. San Francisco, CA: Morgan Kaufmann, 2nd edition, ISBN 1-55860479-0., 1998.

[44] C. Peters. Information-set decoding for linear codes over $\mathbb{F}_{q}$. In PQCrypto 2010, LNCS vol. 6061, pages 81-94. Springer, 2010.

[45] A. E. Pusane, R. Smarandache, P. O. Vontobel, and D. J. Costello, Jr. Deriving good LDPC convolutional codes from LDPC block codes. IEEE Trans. Inform. Theory, 57(2):835-857, 2011.

[46] V. M. Sidelnikov. A public-key cryptosystem based on binary Reed-Muller codes. Discrete Math Appl., 4:191-208, 1994.

[47] V. M. Sidelnikov and S. O. Shestakov. On insecurity of cryptosystems based on generalized Reed-Solomon codes. Discrete Math Appl., 2:439-444, 1992.

[48] J. Stern. A method for finding codewords of small weight. In Coding Theory and Applications, LNCS vol. 388, pages 106-113. Springer, 1989.

[49] H. Stichtenoth. Algebraic Function Fields and Codes. Springer Publishing Company, Incorporated, 2nd edition, 2008.

[50] R. M. Tanner. Convolutional codes from quasi-cyclic codes: A link between the theories of block and convolutional codes. University of California, Santa Cruz, Tech Report UCSC-CRL-87-21, Nov. 1987.

[51] V. Weger. A code-based cryptosystem using GRS codes. Master Thesis at the University of Zürich (Switzerland), 2016.

[52] C. Wieschebrink. Cryptanalysis of the Niederreiter public key scheme based on GRS subcodes. Post-Quantum Cryptography, pages 61-72, 2010. 